PROCEEDINGS OF THE

AMERICAN MATHEMATICAL SOCIETY

Volume 135, Number 8, August 2007, Pages 2433-2442

S 0002-9939(07)09117-4

Article electronically published on April 5, 2007

\title{
ON QUASIPLANES IN EUCLIDEAN SPACES
}

\author{
O. MARTIO, V. M. MIKLYUKOV, AND M. VUORINEN
}

(Communicated by Andreas Seeger)

\begin{abstract}
A variational inequality for the images of $k$-dimensional hyperplanes under quasiconformal maps of the $n$-dimensional Euclidean space is proved when $1 \leq k \leq n-2$.
\end{abstract}

\section{MAin Results}

Below we use the terminology and notation of [7].

Let $\mathbf{R}^{n}$ be the $n$-dimensional Euclidean space, $n \geq 2$, let $f: \mathbf{R}^{n} \rightarrow \mathbf{R}^{n}$ be a mapping of the class $W_{n \text {, loc }}^{1}\left(\mathbf{R}^{n}\right)$, and let $f^{\prime}: \mathbf{R}^{n} \rightarrow \mathbf{R}^{n}$ denote its formal derivative. We write

$$
\left\|f^{\prime}(x)\right\|=\max _{|h|=1}\left|f^{\prime}(x) h\right| .
$$

A homeomorphism $f: \mathbf{R}^{n} \rightarrow \mathbf{R}^{n}$ is called $K$-quasiconformal [10, p. 250-252] if $f \in W_{n, \text { loc }}^{1}\left(\mathbf{R}^{n}\right)$ and

$$
\left\|f^{\prime}(x)\right\|^{n} \leq K J(x, f) \quad \text { a.e. on } \quad \mathbf{R}^{n},
$$

where $J(x, f)=\operatorname{det}\left(f^{\prime}(x)\right)$ is the Jacobian of $f$ at the point $x \in \mathbf{R}^{n}$. The smallest constant $K$ in (1.1) is called the outer dilatation of $f$ and denoted by $K_{O}(f)$. The smallest constant $K \geq 1$ in the inequality

$$
J(x, f) \leq K \ell\left(f^{\prime}(x)\right)^{n}
$$

is called the inner dilatation of $f: \mathbf{R}^{n} \rightarrow \mathbf{R}^{n}$ and denoted by $K_{I}(f)$. Here, $\ell\left(f^{\prime}(x)\right)=\min _{|h|=1}\left|f^{\prime}(x) h\right|$. The quantity

$$
K(f)=\max \left\{K_{O}(f), K_{I}(f)\right\}
$$

is called the maximal dilatation of $f$ [10, Section 14.1].

If $f: \mathbf{R}^{n} \rightarrow \mathbf{R}^{n}$ is quasiconformal, then it is well known that $f\left(\mathbf{R}^{n}\right)=\mathbf{R}^{n}$ and the inverse map $f^{-1}: \mathbf{R}^{n} \rightarrow \mathbf{R}^{n}$ is also quasiconformal in $\mathbf{R}^{n}$ with $K\left(f^{-1}\right)=K(f)$.

Let $d\left(x^{\prime}, x^{\prime \prime}\right)$ be the Euclidean distance for $x^{\prime}, x^{\prime \prime} \in \mathbf{R}^{n}$. We write

$$
B(a, t)=\left\{x \in \mathbf{R}^{n}: d(a, x)<t\right\}, \quad S(a, t)=\left\{x \in \mathbf{R}^{n}: d(a, x)=t\right\} .
$$

Received by the editors April 27, 2004 and, in revised form, July 18, 2005 and November 6, 2005.

2000 Mathematics Subject Classification. Primary 30C62, 30C65.

Key words and phrases. Quasiconformal mapping, quasiconformal curve, quasicircle, uniform domain, quasidisk, quasiplane.

(C)2007 American Mathematical Society 
Let $y=\left(y_{1}, y_{2}, \ldots, y_{n}\right)$ be a point in $\mathbf{R}^{n}$ and $1 \leq k \leq n-2$. Consider a $k$ dimensional plane

$$
\Pi_{0}^{k}=\left\{y=\left(y_{1}, y_{2}, \ldots, y_{n}\right) \in \mathbf{R}^{n}: y_{k+1}=\ldots=y_{n}=0\right\} .
$$

Let $\Pi^{k}$ be a $k$-dimensional surface in $\mathbf{R}^{n}$, i.e., a homeomorphic image of a $k$ dimensional plane. Fix a point $a \in \Pi^{k}$ and $R, 0<R<\infty$. Denote by $\Pi^{k}(a, R)$ a component of $\Pi^{k} \cap B(a, R), a \in \Pi^{k}(a, R)$. We say that a $k$-dimensional surface $\Pi^{k}$ is a $K$-quasiplane, if there exists a $K$-quasiconformal mapping $f: \mathbf{R}^{n} \rightarrow \mathbf{R}^{n}$ such that $f\left(\Pi^{k}\right)=\Pi_{0}^{k}$.

For $n=2$, the following result is well known [1. A curve $\Pi^{1} \subset \mathbf{R}^{2}$ is a $K$ quasiconformal line if and only if there exists a constant $C(K)$ such that

$$
\frac{d\left(\zeta_{2}, \zeta_{1}\right)}{d\left(\zeta_{3}, \zeta_{1}\right)} \leq C(K)
$$

for every triple of distinct points on $\Pi^{1}$ such that $\zeta_{2}$ lies between $\zeta_{1}$ and $\zeta_{3}$.

For more information on quasiconformal lines, we refer to [8], 2, Chapter 14], 9], [11, [18, [12], [17. Quasiplanes $\Pi^{n-1}$ in $\mathbf{R}^{n}$ with codim $\Pi^{n-1}=1$ were considered in [13, 16] and, in the setup of Riemannian manifolds, in 14. Quasiplanes may have a highly complicated structure; see [2, pp. xxii-xxiii]. For instance, it is well known that quasiconformal lines may have Hausdorff dimension $>1$ and hence be non-rectifiable; on the other hand sufficient conditions for the $(n-1)$-rectifiability of quasiplanes $\Pi^{n-1}$ in $\mathbf{R}^{n}$ were given in 13 . Below we consider the case of $K$ quasiplanes in $\mathbf{R}^{n}$ of dimension $1 \leq k \leq n-2$. Very recently, this topic was studied by I. Prause in [19] and 20.

For an arbitrary open subset $\Sigma$ on the sphere $S(a, r) \subset \mathbf{R}^{n}$ we define the quantity

$$
\eta(\Sigma)=\sup _{A} \inf _{\varphi} \frac{\left(\int_{\Sigma}\left|\nabla_{S} \varphi\right|^{n} d \mathcal{H}^{n-1}\right)^{1 / n}}{\left(\int_{\Sigma}|\varphi-A|^{n} d \mathcal{H}^{n-1}\right)^{1 / n}} .
$$

Here the infimum is taken over all functions

$$
\varphi \in W_{n}^{1}(\Sigma),\left.\quad \varphi\right|_{\partial \Sigma}=0
$$

the supremum is taken over all constants $A$, and $\partial \Sigma$ is the boundary with respect to $S(a, r)$.

The quantity $\nabla_{S} \varphi$ is the gradient of $\varphi$ on $S(a, r)$. (The most convenient for our purposes is the definition of $\nabla_{S} \varphi$ on a surface $S \subset \mathbf{R}^{n}$; see, for example, in $\S 2$ of [15.)

The zero boundary values $\left.\varphi\right|_{\partial \Sigma}=0$ are understood in the Sobolev sense, i.e., $\varphi \in W_{n, 0}^{1}(\Sigma)$.

It is clear that

$$
\eta(\Sigma) \geq \inf _{\varphi} \frac{\left(\int_{\Sigma}\left|\nabla_{S} \varphi\right|^{n} d \mathcal{H}^{n-1}\right)^{1 / n}}{\left(\int_{\Sigma}|\varphi|^{n} d \mathcal{H}^{n-1}\right)^{1 / n}} .
$$


Because $\operatorname{dim} S=n-1$, it follows from Sobolev's $C^{0}$-embedding theorem that the right side of (1.3) is $>0$ at least for every $\Sigma$ with a smooth boundary $\partial \Sigma \neq \emptyset$. To find the best $\eta(\Sigma)$ is an open problem.

1.4. Theorem. Let $\Pi^{k}$ be a $K$-quasiplane in $\mathbf{R}^{n}$ with $1 \leq k \leq n-2$. Then for every point $a \in \Pi^{k}$ and all $0<r<R<\infty$, the following relation holds:

$$
\exp \left\{\frac{1}{K_{O}(f)} \int_{r}^{R} \eta(\Sigma(a, \tau)) d \tau\right\} \leq D(n, K)\left(\frac{R}{r}\right)^{n \beta} .
$$

Here $\beta=K^{1 /(n-1)}$ and

$$
D(n, K)=D_{*}^{2 n}, \quad D_{*}=D_{*}(K)=\exp (4 K(K+1) \sqrt{K-1}) .
$$

2. The language of Differential FORMS

Let $1 \leq k \leq n-2$ and let

$$
f=\left(f_{1}, \ldots, f_{k}, f_{k+1}, \ldots, f_{n}\right): \mathbf{R}^{n} \rightarrow \mathbf{R}^{n}
$$

be a $K$-quasiconformal mapping.

Let $\Pi_{0}^{k}$ be a $k$-dimensional plane of the form

$$
\Pi_{0}^{k}=\left\{y=\left(y_{1}, \ldots, y_{k}, y_{k+1}, \ldots, y_{n}\right): y_{k+1}=\ldots=y_{n}=0\right\} .
$$

We will study some properties of the $k$-dimensional surface

$$
\Pi^{k}=f^{-1}\left(\Pi_{0}^{k}\right) .
$$

Fix a point $a \in \Pi^{k}$. For $R>0$ let $\Pi(a, R)$ be the component of $\Pi^{k} \cap B(a, R)$, containing the point $a$ and $\Sigma(a, R)=S(a, R) \backslash \overline{\Pi(a, R)}$.

We consider the differential form of degree $n-1$

$$
\omega=\sum_{i=k+1}^{n}(-1)^{i-k-1} f_{i} d f_{1} \wedge \ldots \wedge \widehat{d f}_{i} \wedge \ldots \wedge d f_{n} .
$$

It is clear that for a.e. $R \in(0, \infty)$, we have

$$
\omega \in W^{1,1}(S(a, R)) \quad \text { and }\left.\quad \omega\right|_{\partial \Sigma(a, R)}=0 .
$$

We have

$$
\begin{aligned}
d \omega & =\sum_{i=k+1}^{n}(-1)^{i-k-1} d f_{i} \wedge d f_{k+1} \wedge \ldots \wedge \widehat{d f}_{i} \wedge \ldots \wedge d f_{n} \\
& =(n-k) d f_{1} \wedge \ldots \wedge d f_{n},
\end{aligned}
$$

and thus,

$$
* d \omega=(n-k) J(x, f) \geq 0 .
$$

For a.e. $R \in(0, \infty)$ we write

$$
\varepsilon_{f}(a, R)=\sup _{\omega_{0}} \int_{\Sigma(a, R)}(* d \omega) d \mathcal{H}^{n-1} / \int_{\Sigma(a, R)}\left|\omega-\omega_{0}\right| d \mathcal{H}^{n-1},
$$

where the supremum is taken over all weakly closed $(n-1)$-forms $\omega_{0} \in W^{1,1}(B(a, 2 R))$ such that

$$
\left.\omega_{0}\right|_{S(a, R)} \in W^{1,1}(S(a, R)) .
$$


Denote

$$
V(a, r)=\int_{B(a, r)} J(x, f) d \mathcal{H}^{n} .
$$

Because $\mathcal{H}^{n}\left(\Pi_{0}\right)=0$ we see that $\mathcal{H}^{n}\left(\Pi^{k}\right)=0$ and for a.e. $r>0$ we have

$$
\mathcal{H}^{n-1}\left(\Pi^{k} \cap S(a, r)\right)=0 \text {. }
$$

Thus,

$$
\int_{\Sigma(a, r)} \omega_{0}=\int_{S(a, r) \backslash \Pi^{k}} \omega_{0}=\int_{S(a, r)} \omega_{0} .
$$

For an arbitrary weakly closed form $\omega_{0}$ [7] and almost all $0<R<\infty$ the following relations hold:

$$
\begin{aligned}
\int_{\Sigma(a, R)}\left|\omega-\omega_{0}\right| d \mathcal{H}^{n-1} & \geq \int_{\Sigma(a, R)}\left(\omega-\omega_{0}\right) \\
& =(n-k) \int_{B(a, R)} J(x, f) d \mathcal{H}^{n}=(n-k) V(a, R) .
\end{aligned}
$$

Here we used that $\left.f_{i}\right|_{\Pi^{k}}=0(i=k+1, \ldots, n)$ and that for the weakly closed form $\omega_{0}$

$$
\begin{aligned}
\int_{\Sigma(a, R)}\left(\omega-\omega_{0}\right) & =\int_{\Sigma(a, R)} \omega \\
& =\int_{\Sigma(a, R)} \sum_{i=k+1}^{n}(-1)^{i-k-1} f_{i} d f_{1} \wedge \ldots \wedge \widehat{d f}_{i} \wedge \ldots \wedge d f_{n} \\
& =\sum_{i=k+1}^{n}(-1)^{i-k-1} \int_{\Sigma(a, R)} f_{i} d f_{1} \wedge \ldots \wedge \widehat{d f}_{i} \wedge \ldots \wedge d f_{n} \\
& =\sum_{i=k+1}^{n} \int_{B(a, R)} d f_{1} \wedge \ldots \wedge d f_{i} \wedge \ldots \wedge d f_{n} \\
& =(n-k) \int_{B(a, R)} J(x, f) d \mathcal{H}^{n} .
\end{aligned}
$$

The relation

$$
\int_{\Sigma(a, R)} f_{i} d f_{1} \wedge \ldots \wedge \widehat{d f}_{i} \wedge \ldots \wedge d f_{n}=(-1)^{i-k-1} \int_{B(a, R)} d f_{1} \wedge \ldots \wedge d f_{n}
$$

is clear in the case of $C^{2}$-smooth maps. In the general case we approximate the homeomorphism $f: \mathbf{R}^{n} \rightarrow \mathbf{R}^{n}$ of class $W_{\text {loc }}^{1, n}\left(\mathbf{R}^{n}\right)$ by smooth maps with the following properties:

i) $f_{s} \rightarrow f$ locally uniformly in $\mathbf{R}^{n}$ as $s \rightarrow \infty$;

ii) $\left\|f_{s}^{\prime}(x)-f^{\prime}(x)\right\|_{L^{n}(D)} \rightarrow 0$ as $s \rightarrow \infty$ for every subdomain $D \Subset \mathbf{R}^{n}$. 
We easily see that such an approximation is possible if we use the familiar technique of approximating Sobolev functions by smoothed averages (see, for example, [6. §4.2.1]). Then we fix arbitrary $r_{1}, r_{2}$ with $0<r_{1}<R<r_{2}<\infty$. Using the formula (2.2) for smooth functions $f_{s}$, we get

$$
(-1)^{i-k-1} \int_{r_{1}}^{r_{2}} d r \int_{B(a, r)} J\left(x, f_{s}\right) d \mathcal{H}^{n}=\int_{r_{1}}^{r_{2}} \int_{\Sigma(a, R)} f_{s i} d f_{s 1} \wedge \ldots \wedge \widehat{d f_{s i}} \wedge \ldots \wedge d f_{s n} .
$$

Since $f_{s} \rightarrow f$ uniformly on $B(a, r)$ and $\left.f_{k}\right|_{\Pi^{k}}=0$, passing to the limit as $s \rightarrow \infty$ we obtain

$$
(-1)^{i-k-1} \int_{r_{1}}^{r_{2}} d r \int_{B(a, r)} J(x, f) d \mathcal{H}^{n}=\int_{r_{1}}^{r_{2}} \int_{\Sigma(a, R)} f_{i} d f_{1} \wedge \ldots \wedge \widehat{d f_{i}} \wedge \ldots \wedge d f_{n} .
$$

We divide both sides of this equation by $r_{2}-r_{1}$. Letting $r_{2} \rightarrow r_{1}$, we see that (2.2) holds for almost all $R>0$.

Thus, (2.1) is proved completely.

Fix $0<\alpha<1$ and choose $\omega_{0}$ such that

$$
\alpha \varepsilon_{f}(a, R) \leq \int_{\Sigma(a, R)}(* d \omega) d \mathcal{H}^{n-1} / \int_{\Sigma(a, R)}\left|\omega-\omega_{0}\right| d \mathcal{H}^{n-1} .
$$

By (2.1) we have

$$
\alpha \varepsilon_{f}(a, R) \leq \int_{\Sigma(a, R)} J(x, f) d \mathcal{H}^{n} / V(a, R)
$$

and setting $\alpha \rightarrow 1$ we obtain

$$
\varepsilon_{f}(a, R) \leq \int_{\Sigma(a, R)} J(x, f) d \mathcal{H}^{n} / V(a, R) .
$$

Since $|\nabla d(a, x)| \equiv 1$ we arrive at

$$
\int_{\Sigma(a, R)} J(x, f) d \mathcal{H}^{n-1}=\int_{S(a, R)} J(x, f) d \mathcal{H}^{n-1}=V^{\prime}(a, R) \geq \varepsilon_{f}(a, R) V(a, R),
$$

which is true for a.e. $R \in(0, \infty)$.

The function $t \mapsto V(a, t)$ is absolutely continuous on $[r, R]$ and solving this differential inequality we have

$$
\exp \left\{\int_{r}^{R} \varepsilon_{f}(\tau) d \tau\right\} \leq \frac{V(a, R)}{V(a, r)} .
$$

We put

$$
l(a, t)=\min _{x \in S(a, t)}|f(x)-f(a)|, \quad L(a, t)=\max _{x \in S(a, t)}|f(x)-f(a)| .
$$

Now,

$$
V(a, r) \geq \Omega_{n} l(a, r)^{n}, \quad V(a, R) \leq \Omega_{n} L(a, R)^{n},
$$


where $\Omega_{n}=\mathcal{H}^{n}(B(0,1))$. Hence,

$$
\frac{V(a, R)}{V(a, r)} \leq \frac{L(a, R)^{n}}{l(a, r)^{n}}
$$

We use the following bound for quasiconformal maps.

2.5. Theorem. Let $f: \mathbf{R}^{n} \rightarrow \mathbf{R}^{n}$ be a K-quasiconformal map. Then for every point $a \in \mathbf{R}^{n}$ and an arbitrary $\rho, 0<\rho<\infty$ the following estimate holds:

$$
\frac{L(a, \rho)}{l(a, \rho)} \leq D_{*}, D_{*}=D_{*}(K)=\exp (4 K(K+1) \sqrt{K-1}) .
$$

For a proof see [2, Theorem 14.8].

Using (2.6), we see that

$$
\frac{L(a, R)}{l(a, r)}=\frac{L(a, R)}{l(a, R)} \frac{l(a, R)}{L(a, r)} \frac{L(a, r)}{l(a, r)} \leq D_{*}^{2} \frac{l(a, R)}{L(a, r)}, \quad D_{*}=D_{*}(K) .
$$

We next show that if $\beta=K^{1 /(n-1)}$, then for $0<r<R$

$$
\frac{l(a, R)}{L(a, r)} \leq\left(\frac{R}{r}\right)^{\beta} .
$$

Indeed, because the case $l(a, R) \leq L(a, r)$ is clear, we may assume that $l(a, R)>$ $L(a, r)$. Applying the well-known formula for the $n$-capacity of a spherical condenser in $\mathbf{R}^{n}$, we obtain

$$
\begin{aligned}
\omega_{n-1}\left(\ln \frac{l(a, R)}{L(a, r)}\right)^{1-n} & =\operatorname{cap}(\bar{B}(f(a), L(a, r)), B(f(a), l(a, R))) \\
& \geq \operatorname{cap}(f \bar{B}(a, r), f B(a, R)) \\
& \geq \frac{1}{K} \operatorname{cap}(\bar{B}(a, r), B(a, R)) \\
& =\frac{1}{K} \omega_{n-1}\left(\ln \frac{R}{r}\right)^{1-n}
\end{aligned}
$$

where $\omega_{n-1}$ is the $(n-1)$-dimensional area of the boundary of $B(0,1)$.

From this inequality we arrive at (2.8). Thus, by (2.7) and (2.8) we obtain

$$
\frac{L(a, R)}{l(a, r)} \leq D_{*}^{2}\left(\frac{R}{r}\right)^{\beta} .
$$

Using (2.7), we now have

$$
\frac{V(a, R)}{V(a, r)} \leq D_{*}^{2 n}\left(\frac{l(a, R)}{L(a, r)}\right)^{n},
$$

and further by (2.8),

$$
\frac{V(a, R)}{V(a, r)} \leq D_{*}^{2 n}\left(\frac{R}{r}\right)^{n \beta} .
$$

The estimates (2.3) and (2.10) imply the following statement. 
2.11. Theorem. Let $\Pi^{k}$ be a $K$-quasiplane in $\mathbf{R}^{n}$ with $1 \leq k \leq n-2$. Then for every point $a \in \Pi^{k}$ and arbitrary numbers $0<r<R<\infty$, we have

$$
\exp \left\{\int_{r}^{R} \varepsilon_{f}(a, \tau) d \tau\right\} \leq D_{*}^{2 n}(K)\left(\frac{R}{r}\right)^{n \beta}, \quad \beta=K^{1 /(n-1)},
$$

for every K-quasiconformal mapping $f: \mathbf{R}^{n} \rightarrow \mathbf{R}^{n}$ such that $\Pi^{k}=f^{-1}\left(\Pi_{0}^{k}\right)$.

\section{Proof of the Main theorem}

Fix a $K$-quasiconformal map $f: \mathbf{R}^{n} \rightarrow \mathbf{R}^{n}$ with $f\left(\Pi^{k}\right)=\Pi_{0}^{k}$. We shall estimate $\varepsilon_{f}(a, R)$ by $\eta(\Sigma(a, R))$. Setting

$$
\begin{gathered}
\omega_{0}=\sum_{i=k+1}^{n}(-1)^{i-k-1} \alpha_{i} d f_{1} \wedge \ldots \wedge \widehat{d f_{i}} \wedge \ldots \wedge d f_{n}, \\
\varphi=\sum_{i=k+1}^{n}\left|d f_{1} \wedge \ldots \wedge \widehat{d f_{i}} \wedge \ldots \wedge d f_{n}\right|^{2}
\end{gathered}
$$

where $\alpha_{i}$ are arbitrary constants, we have

$$
\varepsilon_{f}(a, R) \geq \int_{\Sigma(a, R)} * d \omega / \int_{\Sigma(a, R)}\left|\omega-\omega_{0}\right| d \mathcal{H}^{n-1}
$$

Observe that

$$
\begin{aligned}
& \int_{\Sigma(a, R)}\left|\omega-\omega_{0}\right| d \mathcal{H}^{n-1} \\
& \leq \int_{\Sigma(a, R)} \sqrt{\sum_{i=k+1}^{n}\left(f_{i}-\alpha_{i}\right)^{2}} \sqrt{\sum_{i=k+1}^{n}\left|d f_{1} \wedge \ldots \wedge \widehat{d f_{i}} \wedge \ldots \wedge d f_{n}\right|^{2}} d \mathcal{H}^{n-1} \\
& \leq\left(\int_{\Sigma(a, R)}\left(\sum_{i=k+1}^{n}\left(f_{i}-\alpha_{i}\right)^{2}\right)^{n / 2} d \mathcal{H}^{n-1}\right)^{1 / n}\left(\int_{\Sigma(a, R)}(\varphi)^{n /(2(n-1))} d \mathcal{H}^{n-1}\right)^{(n-1) / n} .
\end{aligned}
$$

It is not difficult to check that

$$
(\varphi)^{n /(2(n-1))} \leq c_{1}(n, k)\left\|f^{\prime}(x)\right\|^{n},
$$

where $c_{1}(n, k)$ is a constant and, in fact,

$$
c_{1}(n, k)=(n-k)^{n /(2(n-1))} .
$$

Indeed, since the form $d f_{1} \wedge \ldots \wedge \widehat{d f}_{i} \wedge \ldots \wedge d f_{n}$ is simple,

$$
\begin{aligned}
& \left|d f_{1} \wedge \ldots \wedge \widehat{d f}_{i} \wedge \ldots \wedge d f_{n}\right|^{2} \\
& =\left\langle d f_{1} \wedge \ldots \wedge \widehat{d f}_{i} \wedge \ldots \wedge d f_{n}, d f_{1} \wedge \ldots \wedge \widehat{d f_{i}} \wedge \ldots \wedge d f_{n}\right\rangle \\
& \leq \prod_{\substack{s=1 \\
s \neq i}}^{n}\left|d f_{s}\right|^{2} .
\end{aligned}
$$


Using the inequality between geometric and arithmetic means we obtain

$$
\left(\prod_{\substack{s=1 \\ s \neq i}}^{n}\left|d f_{s}\right|^{2}\right)^{1 /(n-1)} \leq \frac{1}{n-1} \sum_{\substack{s=1 \\ s \neq i}}^{n}\left|d f_{s}\right|^{2}
$$

and hence,

$$
\left|d f_{1} \wedge \ldots \wedge \widehat{d f_{i}} \wedge \ldots \wedge d f_{n}\right|^{2 /(n-1)} \leq \frac{1}{n-1} \sum_{\substack{s=1 \\ s \neq i}}^{n}\left|d f_{s}\right|^{2} .
$$

Further, for an arbitrary vector $h \in \mathbf{R}^{n}$,

$$
\left|f^{\prime}(x) h\right|^{2}=\sum_{s=1}^{n}\left\langle\nabla f_{s}, h\right\rangle^{2}
$$

and setting $h=\nabla f_{i} /\left|\nabla f_{i}\right|$, we find that

$$
\left|\nabla f_{i}\right|^{2}+\frac{1}{\left|\nabla f_{i}\right|^{2}} \sum_{\substack{s=1 \\ s \neq i}}^{n}\left\langle\nabla f_{s}, \nabla f_{i}\right\rangle^{2} \leq\left\|f^{\prime}\right\|^{2} .
$$

Thus, from (3.4) it follows that

$$
\left|d f_{1} \wedge \ldots \wedge \widehat{d f}_{i} \wedge \ldots \wedge d f_{n}\right| \leq\left\|f^{\prime}(x)\right\|^{n-1}
$$

and (3.2) follows. Now (3.2) yields

$$
\int_{\Sigma(a, R)}(\varphi)^{n /(2(n-1))} d \mathcal{H}^{n-1} \leq c_{1}(n, k) \int_{\Sigma(a, R)}\left\|f^{\prime}(x)\right\|^{n} d \mathcal{H}^{n-1} .
$$

Next we use the inequality

$$
\left(\frac{1}{N} \sum_{i=1}^{n}\left|a_{i}\right|^{t_{1}}\right)^{1 / t_{1}} \leq\left(\frac{1}{N} \sum_{i=1}^{n}\left|a_{i}\right|^{t_{2}}\right)^{1 / t_{2}} \quad\left(t_{1} \leq t_{2}\right)
$$

(see, for example, $4, \S 16$ Chapter I]).

We have

$$
\left(\frac{1}{n-k} \sum_{i=k+1}^{n}\left|f_{i}-\alpha_{i}\right|^{2}\right)^{1 / 2} \leq\left(\frac{1}{n-k} \sum_{i=k+1}^{n}\left|f_{i}-\alpha_{i}\right|^{n}\right)^{1 / n}
$$

and hence,

$$
\left(\sum_{i=k+1}^{n}\left|f_{i}-\alpha_{i}\right|^{2}\right)^{1 / 2} \leq c_{2}(n, k)\left(\sum_{i=k+1}^{n}\left|f_{i}-\alpha_{i}\right|^{n}\right)^{1 / n}
$$


with $c_{2}(n, k)=(n-k)^{(n-2) /(2 n)}$. Now we see from definition (1.2) of $\eta(\Sigma(a, R))$ that

$$
\begin{aligned}
& \int_{\Sigma(a, R)}\left(\sum_{i=k+1}^{n}\left(f_{i}-\alpha_{i}\right)^{2}\right)^{n / 2} d \mathcal{H}^{n-1} \\
& \leq c_{2}^{n}(n, k) \sum_{i=k+1}^{n} \int_{\Sigma(a, R)}\left|f_{i}-\alpha_{i}\right|^{n} d \mathcal{H}^{n-1} \\
& \leq c_{2}^{n}(n, k) \eta^{-n}(\Sigma(a, R)) \sum_{i=k+1}^{n} \int_{\Sigma(a, R)}\left|\nabla_{S} f_{i}\right|^{n} d \mathcal{H}^{n-1} .
\end{aligned}
$$

However, (3.5) implies that $\left|\nabla_{S} f_{i}\right| \leq\left\|f^{\prime}\right\|$ for $i=1, \ldots, n$ and hence,

$$
\sum_{i=k+1}^{n}\left|\nabla_{S} f_{i}\right|^{n} \leq(n-k)\left\|f^{\prime}\right\|^{n}
$$

Thus, we arrive at the estimate

$$
\int_{\Sigma(a, R)}\left(\sum_{i=k+1}^{n}\left(f_{i}-\alpha_{i}\right)^{2}\right)^{n / 2} d \mathcal{H}^{n-1} \leq c_{3}(n, k) \eta^{-n}(\Sigma(a, R)) \int_{\Sigma(a, R)}\left\|f^{\prime}(x)\right\|^{n} d \mathcal{H}^{n-1},
$$

where

$$
c_{3}(n, k)=(n-k) c_{2}(n, k)=(n-k)^{n / 2} .
$$

Substituting these estimates into (3.1), we have the inequality

$$
\varepsilon_{f}(a, R) \geq(n-k)^{-1} \eta(\Sigma(a, R)) \int_{\Sigma(a, R)} J(x, f) d \mathcal{H}^{n-1} / \int_{\Sigma(a, R)}\left\|f^{\prime}(x)\right\|^{n} d \mathcal{H}^{n-1} .
$$

Thus, we obtained

$$
\varepsilon_{f}(a, R) \geq \frac{1}{K_{O}(f)} \eta(\Sigma(a, R))
$$

and using Theorem 2.11, we prove Theorem 1.4,

\section{ACKNowledgments}

The authors are indebted to Istvan Prause for a number of very useful remarks and to the referee for a set of detailed suggestions for the improvement of the text.

\section{REFERENCES}

[1] L. Ahlfors, Quasiconformal reflections, Acta Math. 109 (1963), 291-301.

[2] G.D. Anderson, M.K. Vamanamurthy, and M.K. Vuorinen, Conformal invariants, inequalities, and quasiconformal maps, Canadian Math. Soc., Ser. of Monographs and Advanced texts, A Wiley - Interscience Publication, John Wiley\&Sons, Inc., New-York - Chichester Weinheim - Brisbane - Singapore - Toronto, 1997.

[3] C. Bandle, Isoperimetric inequalities and applications, Pitman Advanced Publishing, Boston-London-Melbourne, 1980.

[4] E.F. Beckenbach and R. Bellman, Inequalities, Springer-Verlag, Berlin - Göttingen Heidelberg, 1961.

[5] M. Bonk and J. Heinonen, Smooth quasiregular mappings with branching, Publ. Math. Inst. Hautes Études Sci. No. 100 (2004), 153-170. 
[6] L.C. Evans And R.F. Gariepy, Measure theory and fine properties of functions, Studies in Advanced Mathematics, CRC PRESS, Boca Raton - New York - London - Tokyo, 1992.

[7] D. Franke, O. Martio, V.M. Miklyukov, M. Vuorinen, and R. Wisk, Quasiregular mappings and $\mathcal{W} T$-classes of differential forms on Riemannian manifolds, Pacific J. Math. 202 (2002), 73-92.

[8] F.W. Gehring, Uniform domains and the ubiquitous quasidisk, Jahresber. Deutsch. Math.Verein, 89 (1987), 88-103.

[9] M. Gromov, Pseudoholomorphic curves in symplectic manifolds, Invent. Math. 82 (1985), 307-347.

[10] J. Heinonen, T. Kilpeläinen and O. Martio, Nonlinear potential theory of degenerate elliptic equations, Clarendon Press, 1993.

[11] S.L. Krushkal, Quasiconformal mirrors, Sibirsk. Mat. Zh. 40 (1999), 880-892.

[12] O. Martio, V. Miklyukov, S. Ponnusamy, and M. Vuorinen, On some properties of quasiplanes, Results Math. 42 (2002), 107-113.

[13] P. Mattila and M.Vuorinen, Linear approximation property, Minkowski dimension and quasiconformal spheres, J. London Math. Soc. (2) 42 (1990), 249-266.

[14] V.M. MikLyukov, On quasiconformally flat surfaces in Riemannian manifolds, Izv. RAN (ser. math.), 67 (2003), 83-106.

[15] J. Simons, Minimal varieties in Riemannian manifolds, Ann. Math., 88 (1968), No. 1, $62-105$.

[16] J. VÄIsÄLÄ, M. VuORINEn ANd H. WAllin, Thick sets and quasisymmetric maps, Nagoya Math. J. 135 (1994), 121-148.

[17] M. Vuorinen, Geometric properties of quasiconformal maps and special functions. I. Quasiconformal maps and spheres. (English. English, Polish summary) Bull. Soc. Sci. Lett. Łódź Sér. Rech. Déform. 24 (1997), 7-22. (see also Errata: "Geometric properties of quasiconformal maps and special functions. I, II, III [Bull. Soc. Sci. Lett. Łódź Sér. Rech. Déform. 24 (1997), 7-22; ibid., 23-35; ibid., 37-58; ]. Bull. Soc. Sci. Lett. Łódź Sér. Rech. Déform. 26 (1998).

[18] M. Vuorinen, O. Martio, and V.M. Miklyukov, On geometric structure of quasiplanes, Proc. of Dept. of Mathematical Analysis and Function Theory, Volgograd State Univ., 2002, 21-31, ISBN 5-85534-531-9.

[19] I. Prause, Flatness properties of quasispheres, Preprint, University of Helsinki 2007.

[20] I. Prause, Distortion of dimension under quasiconformal mappings, Ph.D. thesis, University of Helsinki 2007.

Department of Mathematics and Statistics, University of Helsinki, 00014 Helsinki, FinLAND

E-mail address: martio@cc.helsinki.fi

Mathematics Department, Volgograd State University, 2 Prodolnaya 30, Volgograd 400062, RUSSIA

E-mail address: miklyuk@hotmail.com

Department of Mathematics, University of Turku, 20014 Turku, Finland

E-mail address: vuorinen@utu.fi 